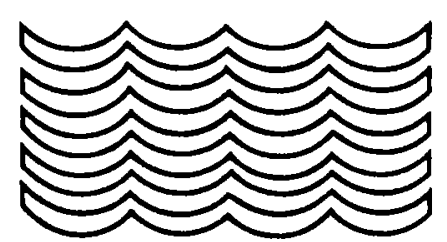

Improved Meteorological Measurements from Buoys and Ships

\title{
A Self-Contained Wind Speed, Direction and Location System for Buoys and Ships in the World Ocean Circulation Experiment
}

by

David S. Hosom, Robert A. Weller and Geoffrey P. Allsup

August 1994

\section{Technical Report}

Funding was provided by the National Science Foundation under Grant No. OCE-87-09614. Approved for public release; distribution unlimited. 


\title{
WHOI-94-19 \\ IMET TR-94-01
}

\section{A Self-Contained Wind Speed, Direction and Location System for Buoys and Ships in the World Ocean Circulation Experiment}

\author{
by \\ David S. Hosom, Robert A. Weller, and Geoffrey P. Allsup \\ Woods Hole Oceanographic Institution \\ Woods Hole, Massachusetts 02543
}

August 1994

Technical Report

Funding was provided by Grant No. OCE-8709614 from the National Science Foundation.

Reproduction in whole or in part is permitted for any purpose of the United States Government. This report should be cited as Woods Hole Oceanog. Inst. Tech. Rept., WHOI-94-19.

Approved for public release; distribution unlimited.

Approved for Distribution:

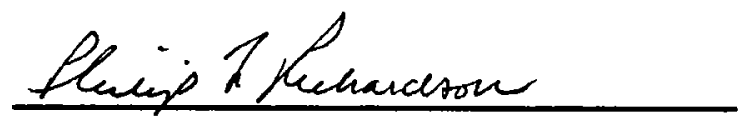

Philip L. Richardson, Chair

Department of Physical Oceanography 


\title{
A SELF-CONTAINED WIND SPEED, DIRECTION AND LOCATION SYSTEM FOR BUOYS AND SHIPS IN THE WORLD OCEAN CIRCULATION EXPERIMENT
}

\author{
David S. Hosom, Robert A. Weller, and Geoffrey P. Allsup \\ Woods Hole Oceanographic Institution \\ Woods Hole, Massachusetts 02543
}

2 August 1994

\section{ABSTRACT}

\begin{abstract}
Knowledge of the absolute wind velocity near the surface of the ocean is a requirement of the world ocean circulation Experiment (WOCE) and other large programs directed towards understanding air-sea interactions and how ocean circulation and climate are interrelated. The measurement is made possible using IMET (Improved METeorology) modules, a next generation meteorological data acquisition system developed as part of the WOCE program. An IMET system consists of a set of intelligent modules for each measurement variable, with data being recorded on a computer, typically PC-based. The IMET wind module includes a propeller for wind speed, a vane and optical encoder for wind direction, a flux gate compass for the north reference, and microprocessor-based electronics for control and data formatting. The IMET Global Positioning system (GPS) module includes a five channel GPS receiver and microprocessor based electronics for control and data formatting. These modules, as part of the complete measurement suite, result in a self-contained system that can make accurate measurements from research ships, drifting and moored buoys, and volunteer observing ships (VOS).
\end{abstract}

\section{Introduction and Background}

The World Ocean Circulation Experiment (WOCE) and other large programs are directed towards understanding air-sea interactions and how ocean circulation and climate are interrelated. During WOCE, moored buoys and ships will provide especially useful platforms from which to make accurate in-situ measurements of the basic observables: sea surface temperature, air temperature, humidity, barometric pressure, wind speed and direction, shortwave and longwave radiation and precipitation. Surface flux estimates can be made from these variables using stability-dependent bulk aerodynamic formulae (Large and Pond, 1981, 1982). Accuracies of $10 \mathrm{~W} \mathrm{~m}-2$ are sought in the estimates of mean values (averaged over monthly and longer time scales) of each of the four components of the total heat flux (sensible, latent, shortwave, and longwave). Accuracies of approximately $1 \mathrm{~mm}$ per day are sought in evaporation and precipitation; $10 \%$ or $0.1 \mathrm{~Pa}$ is sought in stress. 
Key to the measurements and essential for the estimation of the fluxes with the bulk formulae is accurate determination of the absolute wind velocity. This requires two sets of measurements including accurate relative wind speed and direction from the wind sensor, and platform speed and direction from navigation equipment. The wind sensor provides direction relative to a magnetic (flux gate) compass, and this must be corrected for magnetic variation. This is best done in post-processing if the platform travels through magnetic variations of significant magnitude. Platform speed and direction come from the GPS unit. An alternative for some ship installations is to remove the compass from the wind sensor, align the wind sensor to the ship, and then use the ship gyro compass for heading. This results in not having to correct the magnetic variation but introduces alignment errors.

The IMET system is a next-generation meteorological data acquisition system developed for use on buoys and ships. It is modular and intended to have the flexibility to be easily adapted for specific situations. The improved sensors, electronics and intelligent sampling techniques, which are now commercially available, can be used to collect high-quality meteorological data. Work on IMET began in November 1987, when long lead time equipment development, a three year program was in preparation for the World Ocean circulation Experiment, was begun. The goal of this was improved meteorological measurements from ships and buoys. This effort was completed by finalizing the IMET configuration for UNOLS (University National Oceanographic Laboratory System) ships and documenting the system for broad community use. The documentation is commercially available.

The IMET System provides measurements of wind speed and direction relative to magnetic north from the IMET Wind Module and position from the IMET GPS Module without the necessity of interfacing to other ship or buoy systems. The IMET GPS Module in now in a prototype status and requires field testing before becoming commercially available. When both units are commercially available, easy installation will be feasible on ships such as vos or on moored or drifting ocean buoys. The combination of wind speed, direction and position as a function of time in a common data set results in accurate absolute winds and provides a record of platform position and velocity. Ships travel from place to place as do drifting buoys. Moored buoys also move about their anchor position. Determination of wind and currents with correction for platform motion is essential to the calculation of the air-sea fluxes. This paper will briefly describe the IMET system and describe in more detail the IMET Wind Module and the IMET GPS Module. 


\section{IMET System Description}

The IMET system (see Hosom et al,, 1994 for more detail) consists of a central data recorder and a set of distributed sensor modules (Figure 1). Each of the sensor modules is "intelligent", capable of standing alone and supplying both raw and calibrated data via RS-232 or RS-485. On a buoy or on a ship a set of such modules is mounted and linked to the data recorder via a 4-wire RS485 network plus power. Signal conditioning, averaging, and some computation is done within each module. The data recorder carries out the tasks of acquiring data from sensor modules, storing the data, and formatting and sending a data string to a satellite telemetry module (ARGOS).

A large number of different sensors were tested over the past several years to find those that meet the desired accuracies, resolutions, and reliability at reasonable costs. Based on these tests, a group of eight "strawman sensors" were selected to be included into operational systems. These strawman sensors were built into the intelligent modules.

An intelligent module has all of the sensor specific functions of the data acquisition system physically and functionally packaged together with communications to the main recorder on a (RS485) network. These functions include the sensor(s), signal conditioning, data conversion, calibration, processing algorithms, formatting for recording, and network communications. The manufacturing history and identification information, the calibration constants, and data format specifications are all stored in the module. This information is available from the module using the network communications command structure. The network communications permit the main logger to request raw and/or calibrated data. In the test or calibration operation, the network communications permit operation of the module while not attached to the main recorder. Since each module is sampling and processing at the same time, data are collected and reported in parallel instead of in sequential time. Sequential sampling results in undesirable time offsets in different variables. The calibration information can be collected from each module at the system initialization time and stored in the main data recorder, thereby insuring that the data and their calibration are together. This reduces labor and errors in the post processing as well as reducing the complexity of the data recorder. Changing or adding modules in the field is fast and simple, consisting of mounting the physical package, connecting 4 wires ( $+12 \mathrm{vdc}$, common, +RS485, and -RS485) and changing an ASCII configuration file on the main recorder computer. Each module is housed in a plastic, watertight 4.5-inch housing with a watertight stuffing tube for the cable. Each autonomous module package 
acquires and processes meteorological measurements, and transmits data digitally over an RS-485 link to a central data recorder/controller upon command. An RS-232 link provides easy lab interface for development activities. This modular approach, however, increases the cost, power consumption, and number of components in the system. A tradeoff has to be made between these factors and the requirement for system flexibility. Further details of module configuration and operation have been delineated by Allsup (1990).

The data recorder for a ship is a PC-based system that can operate on a widely varying number of computers. The data recorder used at the Woods Hole Oceanographic Institution (WHOI) for a buoy is a very low-power computer called a TattleTale 7. The communications protocol to the intelligent modules is RS485. The intelligent modules are interrogated on a predetermined data cycle, nominally $1.0,3.75,7.5$ or 15 minutes (with data already processed and averaged within the module). The data are collected and stored on a disk. Magnetic hard disks have been used with 120 megabyte capacity on the buoy unit and optical disks with 400 megabyte capacity on the ship unit. The ship data are stored in netCDF (network Common Data Format), which permits use of standard data base, graphics, display, and editing software for ease of processing. The data recorder computer averages several-hour blocks of the various meteorological and calculated flux data and communicates the data for telemetery via the ARGOS module.

The IMET ship system uses a standard MS-DOS 386 PC and can collect data, store it, and provide real time display as well as immediate data editing and hard copy for reports and review. Since the software is written in ' $C$ ', it would be straightforward to have it implemented in Xenix or Unix as well as MS-DOS. For reliability it was decided that the IMET systems on WHOI ships be self-contained and communicate with other ship systems via ethernet.

The IMET buoy data recorder/controller used on early deployments was a DOS-based low-power unit called LOPACS. The LOPACS was programmed in ' $\mathrm{C}$ '. This has been replaced with a commercially available unit called a TattleTale7, which also is programmed in ' $\mathrm{C}$ '. The unit requires very low power and is packaged in an underwater housing for environmental protection. The software is similar to the ship software but with an emphasis on unattended operation for extended periods. 


\section{IMET Find Module}

The IMET module consists of a propeller, vane encoder, and flux gate compass to measure vector-averaged wind speed to the east and north, and outputs these variables once per sample period along with the average, minimum and maximum wind speed, last compass reading and last vane reading. Note that since the data are combined with ocean current data for later analysis, the direction convention chosen is oceanographic, opposite to the meteorological standards of wind from a direction. Figure 2 is a photograph of the complete wind module assembly.

An R. M. Young Model 5103 propeller and vane unit and a KVH Model C100 compass were chosen. The R. M. Young propeller was chosen because of its response characteristics and because it had already been used successfully on oceanographic buoys. Cup anemometers overspeed in the field, measuring mean winds that are 5-10\% too high. (See Weller et al., 1990 for a summary of cup and propeller anemometer errors). Sonic anemometers require more power and have not as yet demonstrated their reliability in unattended buoy and ship installations. Propeller/vane sensors are not subject to overspeeding errors and are believed to provide mean winds within 5\% of the true mean. The propeller and vane have stainless steel bearings installed for longer life operation at sea. The KVH flux gate compass was chosen because of its accuracy and commercial availability. The KVH compass is a microprocessorbased unit that incorporates a field compensation capability to correct for fixed field variations. Associated with the propellervane sensor and compass are the electronics modules.

a. Hardware - Mechanical

The IMET wind speed and direction module uses an R. M. Young Model 5103 "Wind Monitor" propeller and vane unit. The unit is assembled onto a custom endcap and stem at the factory so that it interfaces to the standard IMET housing. The internal coil produces 3 pulses per revolution and has a calibration constant of 0.098 meters of wind per pulse. The lower threshold of the unit is about 0.5 meters per second, and the upper limit of the unit is at about 60 meters per second. Figure 3 is an expanded drawing of the component parts.

Wind direction is the direction that the wind is going to, and is the sum of the clockwise compass azimuth measured from compass north to the housing index and the clockwise vane direction measured from the housing index line. The standard vane readout on 
the Model 5103 is a potentiometer which gives 0 to 355 degrees of coverage. This has been replaced in the IMET unit with a 9-bit absolute optical encoder unit to encode the vane position. This provides a full 360 degrees of coverage as well as providing better long term reliability.

The (magnetic) north reference, a $\mathrm{KVH}$ Industries Inc. flux gate compass (Model C100), interfaces to the module electronics via 2 serial port lines. The $\mathrm{C} 100$ has a gimballed sensor with $+/-45$ degrees of tilt capability, and has an autocompensation table stored in the processor memory for magnetic variations in the compass. A field adjustment to the factory compensation can be made after the compass is installed in the system. This capability is now being utilized for the completed wind speed and direction module and in later versions will be set up to be carried out on the platform (ship or buoy). The c100 compass also is capable of being programmed by the user for different output formats including serial RS232, digital (BCD), linear analog or sine/cosine analog.

The housing of the IMET Wind Module is made of white PVC pipe, with unique locking spline attachments between the tubes and endcap sections. The tube has one half of a cylindrical groove and the endcap has the other half of the groove. A 0.125-inch-diameter delrin spline then fits through an opening in the side of the tube and slides around the circumference of the tube to lock the two pieces together. See Figure 4 for a detail of this. An o-ring inside the spline provides a watertight seal for the interior.

\section{b. Hardware - Electronics}

The module electronics uses three boards, the SCM51 (Sensor Control Module), the PWCOM (Power and Communications), and the WNDSPVN (Wind speed and Vane) board. The first two of these are standard IMET module boards and the last is a custom board with a Gray to binary interface for the encoder and a counter for the wind speed pulses. The SCM51 processor board uses an $80 \mathrm{C} 32$ CMOS microcontroller and 24 bits of parallel I/O. The operating software is written in resident Intel BASIC-52, with additional BASIC language extensions in the firmware PROM. A special interrupt routine in this PROM implements the standard command and communications format used by all modules, and a (BASIC) timer interrupt is used to effect a simple watchdog failsafe control. one custom command permits serial communications and control of the flux gate compass. The BASIC-52 operating software is stored in PROM for automatic run on power up. The present software has been written in ' $C$ ' for improved reliability. See Figure 5 for a block diagram of the wind module. 
The power and communications (PWCOM) board provides RS485 as well as RS232 communications, plus power regulation and control circuitry for the standard boards and optional additional boards.

A serial EEPROM for important but modifiable parameters resides on the PWCOM board, with a two-wire interface to the SCM51.

The custom wind speed and vane (WNDSPVN) board has several functions. The vane is sampled at once per second. It has a power switch for the vane encoder LEDs that is turned on by the SCM51 for a few milliseconds while the encoder Gray code is sampled into a storage buffer via a 9-bit-Gray-to-binary conversion circuit and then the LEDs are turned off. It also has a pulse squaring circuit for the low level sine wave signal generated by the wind speed propeller which is counted and stored into a 16-bit buffer. When the data are read out, the vane and counter buffers are gated out (as high and low bit sets) onto the 8-bit data bus to the SCM51.

c. Software and Firmware

To insure that independently developed modules are compatible, a BASIC-52 software package has been created for use as a starting point. This software provides a common core of support for the standard-IMET communications command set, manufacturing and calibration information, and simple fail-safe and error recovery methods. The software is easily modified with new main loop code specific to a particular sensor being written and debugged interactively. Both software and hardware enhancements can be added, using only a PC with a text editor and a terminal program having ASCII transfer capability. The standard module software supports a modified SAIL (IEEE 997-1985) protocol communications scheme. All modules have unique ID's; an ID preceded by a \# sign is required with each command. All standard commands are single characters as outlined below. Command processing commences immediately upon receipt of the command character. From the table then, the command to the IMET module to transmit (output) calibrated wind data would be [\#WND01C].

IMET Sensor Module standard Command set:

A - Address acknowledge

C - output calibrated data

E - Output EEPROM constants

$\mathrm{H}$ - Help menu

I - Report ID information

$R$ - output raw data

$T$ - Enter test mode

U - Update EEPROM constants 
The main loop area of the standard software has only one predefined task. It must continually update a pair of communications output buffers which contain the formatted response to the $C$ and $A$ commands. The sampling algorithm, acquisition hardware control, and computation of calibrated values were programmed in BASIC but are now in "C". The BASIC standard and custom commands are resident in the firmware EPROM. A fail-safe timeout is implemented so that if the program hangs up, the module will restart itself as it does on power-up. The typical measurement cycle, set at 15 seconds, can be vector-averaged over a range of values $(1.0,3.75$, 7.5 or 15 minutes). The cycle goes through the following steps:

1. Start 15 second cycle.

2. Measure current compass direction.

3. Measure the vane angle every 1.0 second.

4. At the 15 second time,

- calculate wind speed from counts divided by time.

- calculate wind direction, equal to average vane plus compass.

- calculate north and east wind unit vectors.

- calculate the vector average over the specified interval and put in output buffer.

5. Return to 1 (start).

6. Output routine:

Wind module outputs a measurement that is vector averaged over the specified interval $(1.0,3.75,7.5$, or 15 minutes). Wind East, Wind North, Scalar Average, Max (15 second) Speed, Min (15 second) Speed, Last Compass and Last Vane.

\section{d. Specifications}

The IMET wind speed and direction unit thus has the following specifications:

1. Wind Speed.

0.5 to 60 meters/second.

Propeller has a calibration constant of 0.098 meters of wind per pulse.

There are 3 pulses per propeller revolution. 
2. Wind Direction.

Vane encoder is 9 bits for a resolution of 0.7

degrees.

The flux gate compass has a resolution of

0.1 degrees and an accuracy of 0.5 degrees.

There are a full 360 degrees of range with no

deadband.

Direction is averaged over the sample time.

3. Physical.

Housing Diameter is $11.4 \mathrm{~cm}$ or 4.5 inches.

Overall Height is 1.1 meters or 44 inches.

Overall Length is $55.9 \mathrm{~cm}$ or 22 inches.

Weight is $6.4 \mathrm{~kg}$ or 14 pounds.

4. Electrical.

Power supply is +12 vdc to +15 vdc.

Current is $30 \mathrm{ma}$ average, $300 \mathrm{ma}$ peak.

Power is 360 milliwatts average.

5. Communications.

RS232 or RS485.

\section{IMET Magellan GPS Module}

The IMET Magellan GPS module is a flexible unit for high accuracy location via satellites of the Global Positioning System. A Magellan OEM GPS Receiver board was chosen due to its low cost and ease of use. IMET-compatible communications on an RS-485 communications loop allow easy integration with standard IMET sensor suites controlled by a central data logging and control computer. Significant features include internal EEPROM storage of almanac data, and commands to allow direct access to the Magellan GPS Receiver board for test purposes. See Figure 6 for a photograph of the GPS module. This module is in a prototype status and is due for field testing on a buoy of opportunity. After field testing the module will be transferred for commercial production and availability.

\section{a. GPS Module Operation}

Upon powering up the IMET GPS module, the Magellan GPS Receiver must be initialized correctly to begin receiving satellite data and providing position and time information. If no almanac is available in EEPROM, additional time will be required to acquire an almanac. The Magellan GPS Receiver only needs to be initialized 
within about 200 miles of the actual present location. Once a position is acquired, the position update rate of the Magellan GPS Receiver is approximately once per second. The IMET GPS module may be interrogated as needed for position, time, and course, if applicable.

b. Hardware - Electronics

The electronics module uses three boards, including the SCM51 and PWCOM described above for the IMET Wind Module, as well as a UART board for communications with the Magellan OEM GPS board. The UART board is a custom board for this module, containing a UART, RS-232 level shifter, and power control FET for the Magellan GPS board.

The SCM51 processor board uses an $80 \mathrm{C} 32$ CMOS microcontroller and 24 bits of parallel I/O. The operating software is written in resident Intel BASIC-52, with additional BASIC language extensions in the firmware PROM. A special interrupt routine in this PROM implements the standard command and communications format used by all modules, and a (BASIC) timer interrupt is used to effect a simple watchdog fail-safe control.

The PWCOM power and communications interface board provides switched system power, RS-485 as well as RS232 communications, power down and reset capabilities, and serial EEPROM. A primary power kill switch is provided which allows the SCM51 to "pull its own plug" and power down the system. All primary power may be restored by cycling external 12 volt power. Alternatively, since the PWCOM board remains operative, RESET of the primary power may be caused by a communications "break" condition of at least $250 \mathrm{~ms}$. A $2 \mathrm{~K}$ serial EEPROM provides non-volatile, secure storage of operating parameters, such as a flag indicating automatic load of an almanac in EEPROM to the Magellan GPS board at power-up time. This and all other parameters may be modified without program change.

The GPS UART board provides an RS-232 compatible serial interface, as well as ON/OFF power switching, for the Magellan GPS Receiver board. It contains an SCC2691 UART running at 9600 baud and a MAX233 for RS-232 level shifting, plus the necessary 'glue' to attach the UART to the $80 \mathrm{c} 32$ processor bus. Additionally, GPS Receiver board power (Main and ON/OFF) is routed through the board, with FET switching of the ON/OFF line controlled by the $80 \mathrm{c3} 2$. 
c. Firmware

The IMET GPS Module operating firmware is stored in a single $32 \mathrm{~K}$ EPROM. Operation is compatible with standard IMET sensor modules--in fact the primary function of the GPS module firmware is as an IMET-compatible interface for the Magellan OEM GPS receiver board. Unlike IMET sensor modules, a more extensive command set of variable length commands is required to operate the GPS Receiver board. As with the standard sensor module firmware, a modified SAIL (IEEE 997-1985) communications protocol is supported--a unique ID preceded by a \# sign is required with each command. Since variable length commands are required, command processing commences only upon receipt of a terminating carriage return character (ASCII oDh). Commands are parsed from a table, shown below:

GPS Module command set ( ... indicates further characters follow):

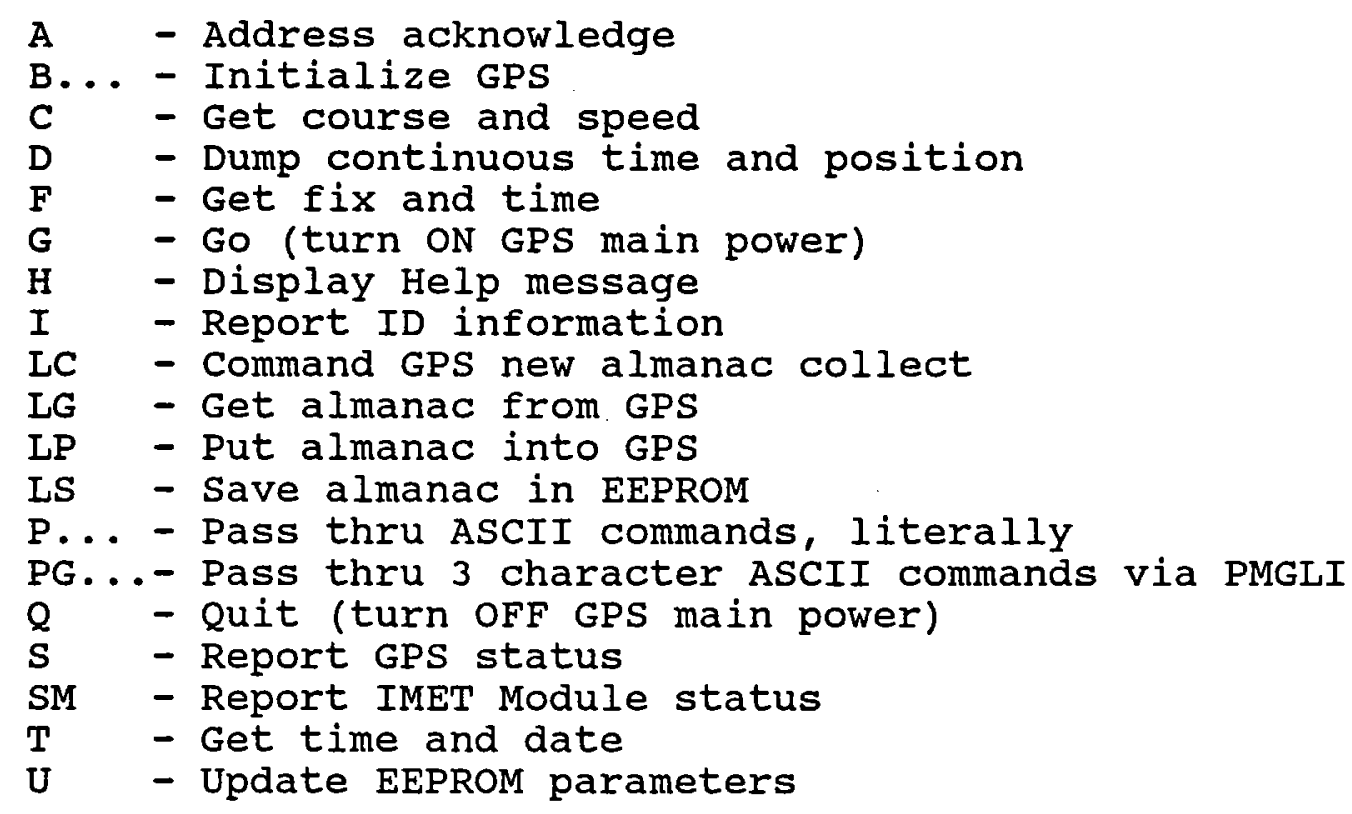

The $A, H, I$, and $U$ commands provide standard responses as with al1 IMET modules. The $G$ and $Q$ commands are self-explanatory. The bulk of the non-standard commands is concerned either with sending information to the Magellan GPS board in the correct NEMA compatible command protocol, or requesting information from the GPS board via the same protocol, and formatting the responses into usable format for return to the IMET data recorder. If Magellan GPS board main power is ON, the GPS board will continuously acquire satellites and update its fix. No intervention by the IMET module 
is needed except when a command request requires access to the Magellan GPS board. In fact, when no requests to the IMET GPS Module are being made, the microcontroller enters an idle state to save power. However, if the Magellan GPS receiver board main power has been OFF but an almanac and recent position (within 200 miles) are in standby-powered memory, the time to acquire a $f i x$ is approximately 2 minutes. The bulk of the module power consumption is from the Magellan GPS board, requiring careful power management by the data recorder system in buoy applications.

d. Hardware - Mechanical

The IMET Magellan GPS module uses a 5-channel OEM GPS receiver available from Magellan systems Corporation, with improved performance, both electrical and operational. The unit comes with a small remote antenna with integral pre-amplifier, plus miscellaneous antenna mounting hardware. The 2 board receiver set is mounted in modified IMET chassis box, attached to the top of the standard IMET chassis box containing the SCM51, PWCOM, and UART boards. The housing of the IMET Magellan GPS module is made of the same white PVC described for the IMET Wind module above. The antenna is mounted to the top end cap of the module, with the antenna cable entering the housing through the bottom end cap opposite the IMET module power and communications cable.

e. Specifications

1. Operational Characteristics:

Position update rate:

Time to first fix (2D):

1 second

2.5 minutes

2. Position Accuracy :

(subject to DoD 'selective availability' policy)

Position: 25 meters in 2D RMS

Velocity: 0.3 knots RMS

3. Physical:

Housing diameter: $\quad 11.4 \mathrm{~cm}$ or 4.5 inches

Overall length with antenna: $67.3 \mathrm{~cm}$ or 26.5 inches

Weight: $\quad 4.5 \mathrm{~kg}$ or 10 pounds 
4. Electrical: Power Supply:

5. Communications:

$$
\begin{aligned}
& +12 \text { to }+15 \text { VDC } \\
& \text { a } 12.7 \mathrm{ma} \text { (GPS OFF) } \\
& \text { a } 347 \mathrm{ma} \text { (GPS ON) }
\end{aligned}
$$

RS232 or RS485

\section{Discussion / summary}

In developing the IMET wind velocity and navigation modules our goal was to address two problems: 1) accurate absolute winds from ships; and, 2) a combination of good wind velocity and buoy position time series from surface moorings.

our experience with wind sensors at sea led to the choice of the R. M. Young propeller/vane sensor. With care taken to ensure that corrosion-resistant bearings were installed and with aluminum substituted for plastic in the mounting base for greater strength, we have had good success with the propeller/vane sensor. The vane encoder has resulted in increased stiffness in the assembly that improves by itself with time. An initial powered "run-in" should be considered for future assemblies. IMET wind modules have been installed on several research ships and buoys. The ships include Oceanus, Knorr, Thompson, and Melville. The buoys were used on projects including Subduction, the Vancouver acoustics experiment, the Atlantic acoustic experiment, TOGA-COARE (Tropical Ocean and Global Atmosphere/Coupled Ocean Experiment), and SWADE (Surface Wave Dynamics Experiment). Buoys with IMET systems are planned for GLOBEC (Global Ocean Ecosystems Dynamics), and the experiment in the Arabian Sea.

Buoy operation has special low-power requirements. The current buoy installation uses six, 20-watt solar panels, with regulators charging six, 80-amp-hour, 12-vdc lead-acid batteries. This provides approximately 5 watts long term average power under normal conditions. When GPS, ARGOS, and aspiration of temperature modules are required, the current power system is marginal. One way to lower power consumption on the standard IMET modules is to lower the clock rate on each module processor. However at a lower clock rate, the BASIC program runs too slow to process the data properly. Modules being used on the TOGA Program have been programmed in ' $C$ ', which runs (very much) faster than BASIC, with a reduction in power for the IMET processor/power communications boards to about $1 / 3$ of the standard specification. Selective ontimes are still required for reduction of power on units like the GPS. 
The KVH C100 compass has provided an excellent magnetic north reference. Buoy spin tests made near the WHOI clark Building show a typical flux gate sine error curve within the few degree tolerance expected. The compass has the capability for interactive field compensation to minimize the effects of fixed nearby magnetic fields. This capability is used on the module by itself at present. Future models of the wind sensor can have the capability of being compensated in place, thereby removing the effects of the ship or buoy for very critical experiments. Buoy applications demand lower power consumption. The compass can be duty-cycled for lower average power.

The GPS module was initially developed around the one-channel Magellan OEM GPS Receiver board set. The ship system works well with this unit since the power can be on continuously. Having the GPS data included with the other meteorological data from the ship has become a firm requirement by the ship operators as well as the scientific users. The buoy system, however, had a problem with power cycling since, upon loss of signal, the unit had to reinitialize. We now use a Magellan 5-channel OEM GPS Receiver. The 5-Channel Receiver requires 30 seconds to re-acquire if ephemeris is less than 2 hours old, 75 seconds otherwise. Also, the 5-channel GPS receiver only consumes 2.4 watts compared to the nearly 4 watts with the single channel receiver. With this configuration, we have a GPS module suited for use on moored buoys. The unit is set up to respond to data recorder requests with approximately 12 minutes of averaged position data. This minimizes the GPS jitter. By programming the data recorder to request data every four hours on a moored buoy, a favorable trade off between power and data rate is achieved. Besides allowing us to correct near-surface current meter records for platform motion, this will also permit use of the less expensive (data only, no position information) class of service from ARGOS. In the future, the lower power consumption of the new GPS module should also allow us to design a stand alone (no connections to ships power or ships navigation) IMET system for use on vessels of opportunity. It has been difficult to balance the need to keep power consumption low against the need to ensure good position information.

\section{Acknowledgements}

The IMET system was part of the Long-Lead Time Development Activity for the World Ocean Circulation Experiment (WOCE) supported by Grant No. OCE-8709614 from the National Science Foundation Ocean 
Science Division. The following people contributed to the program in various ways and were critical to its success: G. Crescenti, $C$. Grant, A. Hinton, N. McPhee, R. Payne, and K. Prada.

\section{REEERENCES}

Allsup, G., 1990: A Standardized Electronics Package for IMET Sensor Development. IEEE Proceedings OCEANS 190, Washington D.C., September 24-26, pp. 164-168.

Hosom, D. S., Weller, R. A., Payne, R. E., and Prada, K. E., 1994: The IMET (Improved METeorology) Ship and Buoy systems. J. Atmos. Oceanic Tech., accepted.

Large, W. G. and S. Pond, 1981: Open Ocean Momentum Flux Measurements in Moderate to strong Winds. J. Phys. Oceanogr., $11,324-336$.

Large; W. G. and S. Pond, 1982: Sensible and Latent Heat Flux Measurements Over the Ocean. J. Phys. Oceanogr., 12, 464-482 .

Weller, R. A., D. L. Rudnick, R. E. Payne, J. P. Dean, N. J. Pennington, and R. P. Trask, 1990: Measuring Near-surface Meteorology over the Ocean from an Array of Surface Moorings in the Subtropical Convergence Zone. J. Atmos. Oceanic Tech., $7(1), 85-103$. 


\section{IMET System Block Diagram}

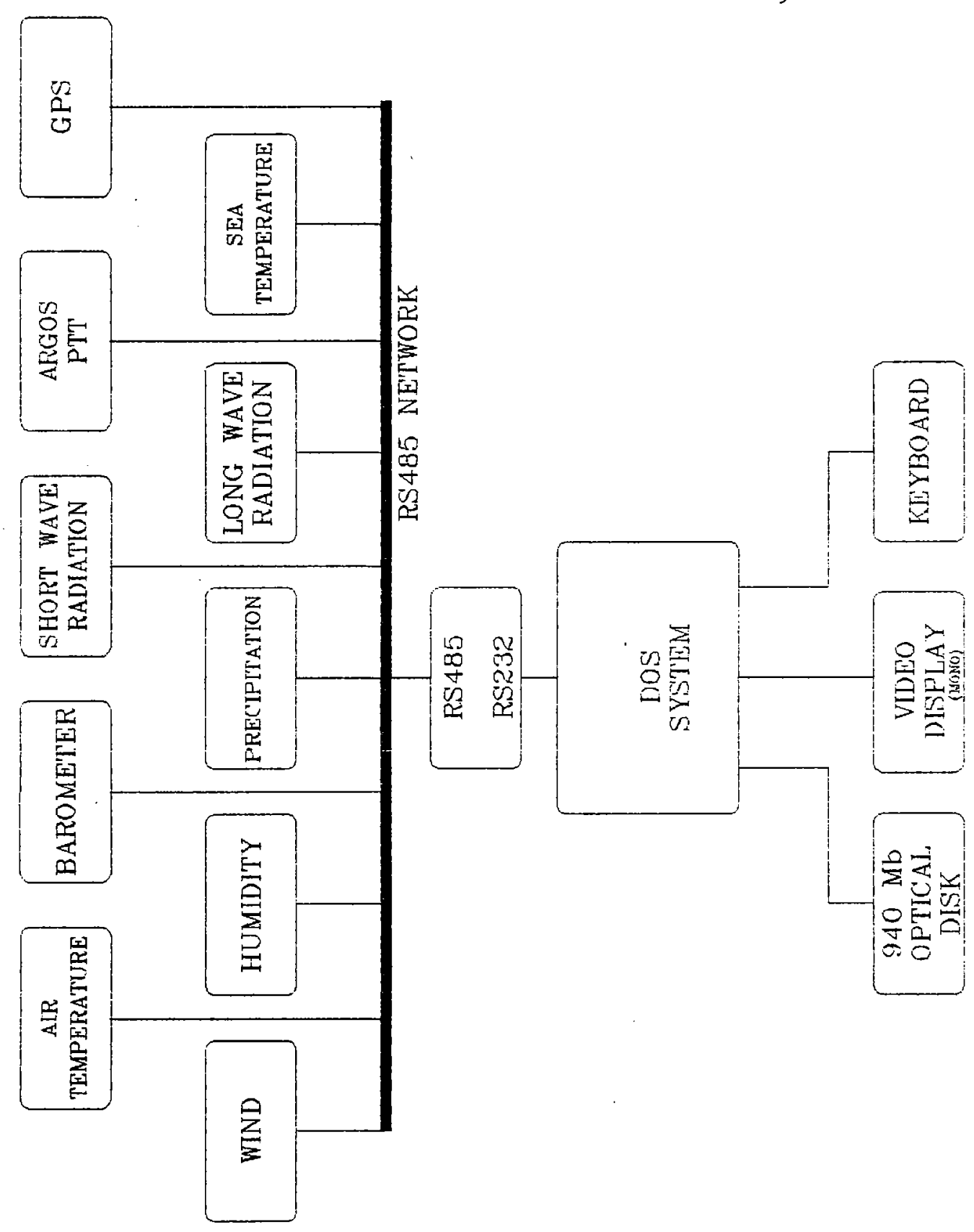




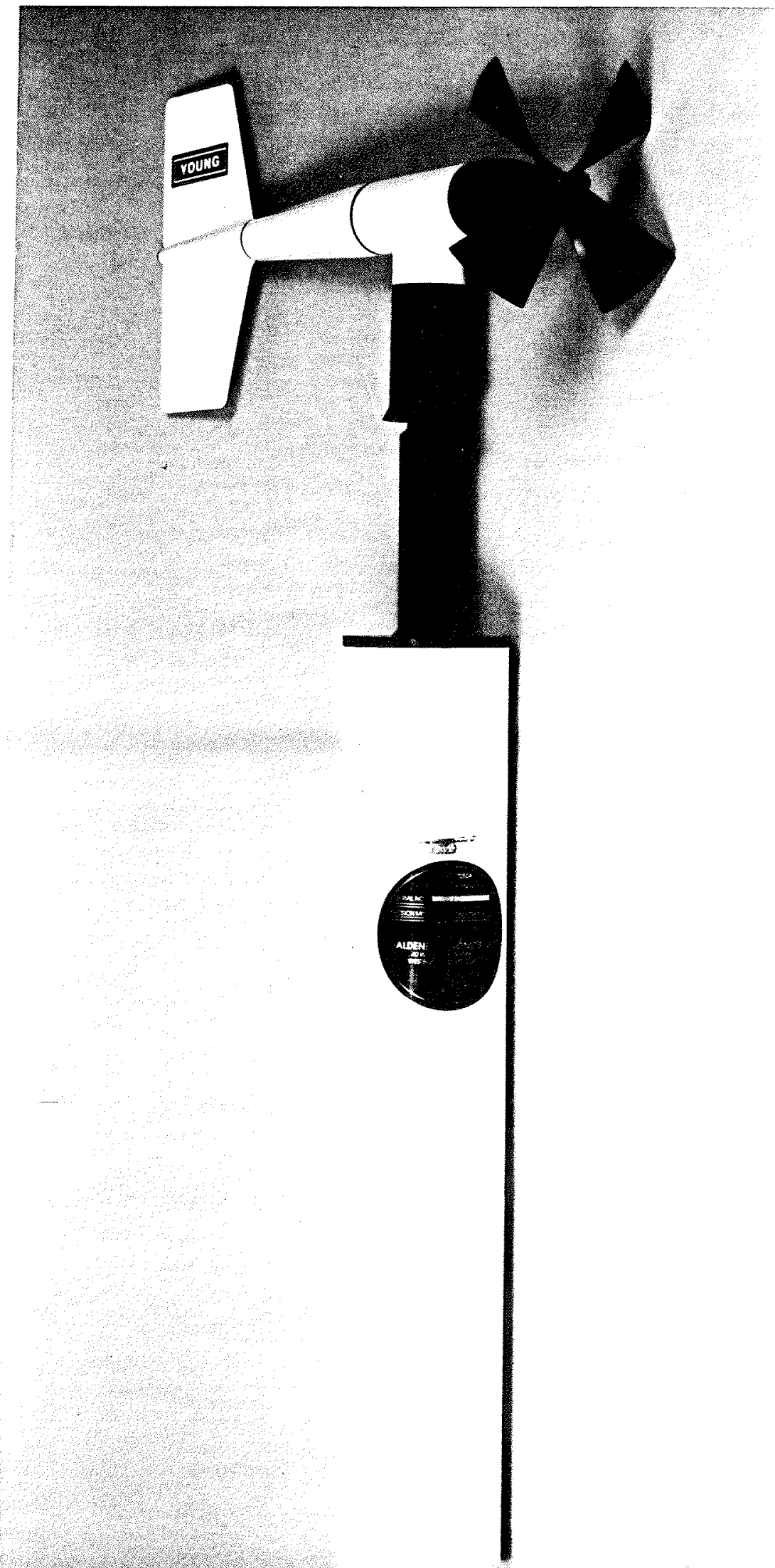

Photograph of

IMET Wind Module

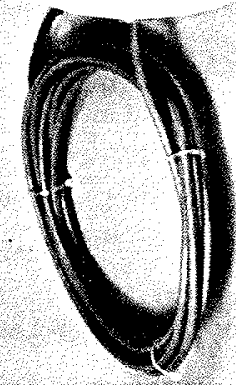



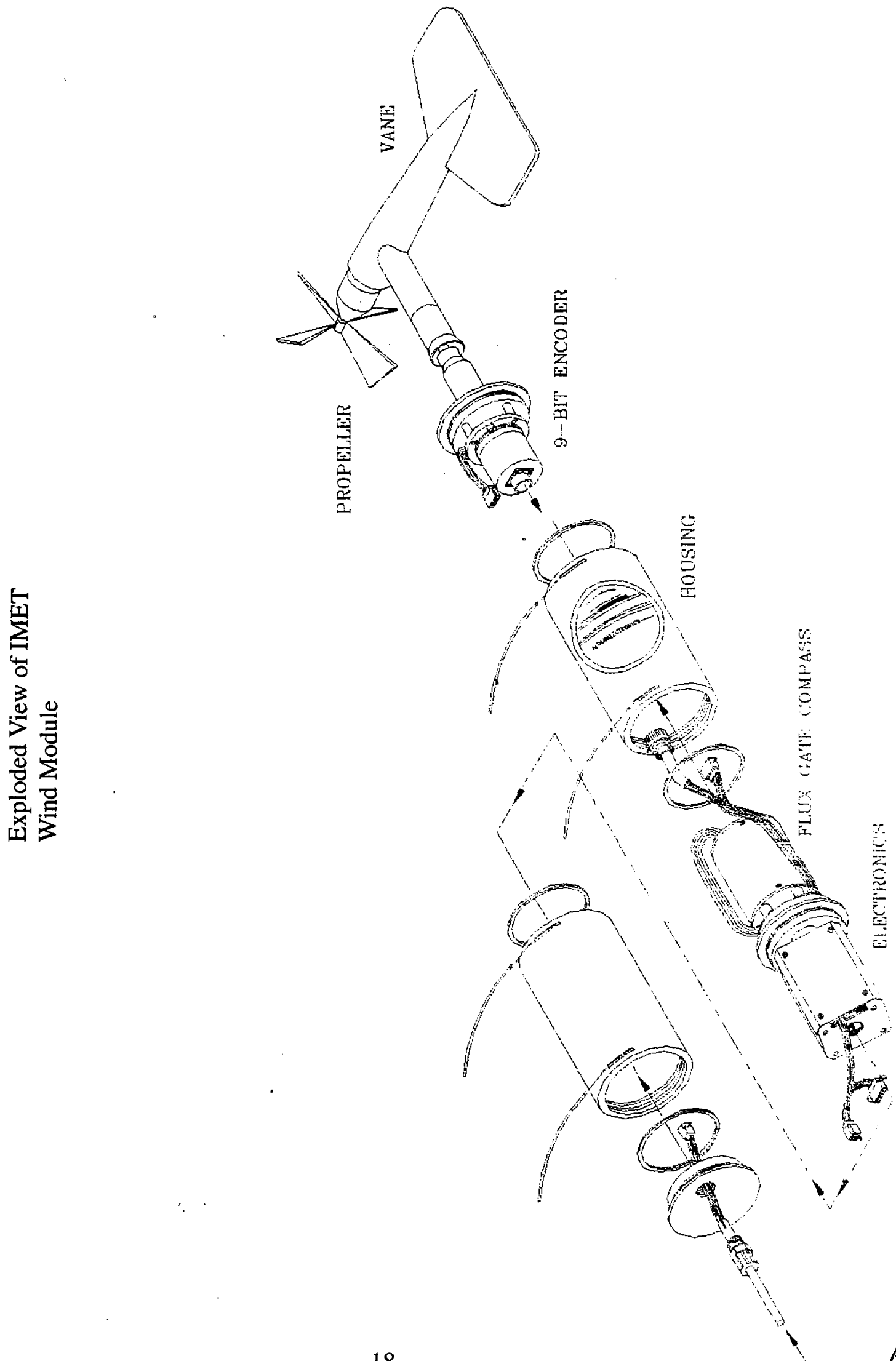


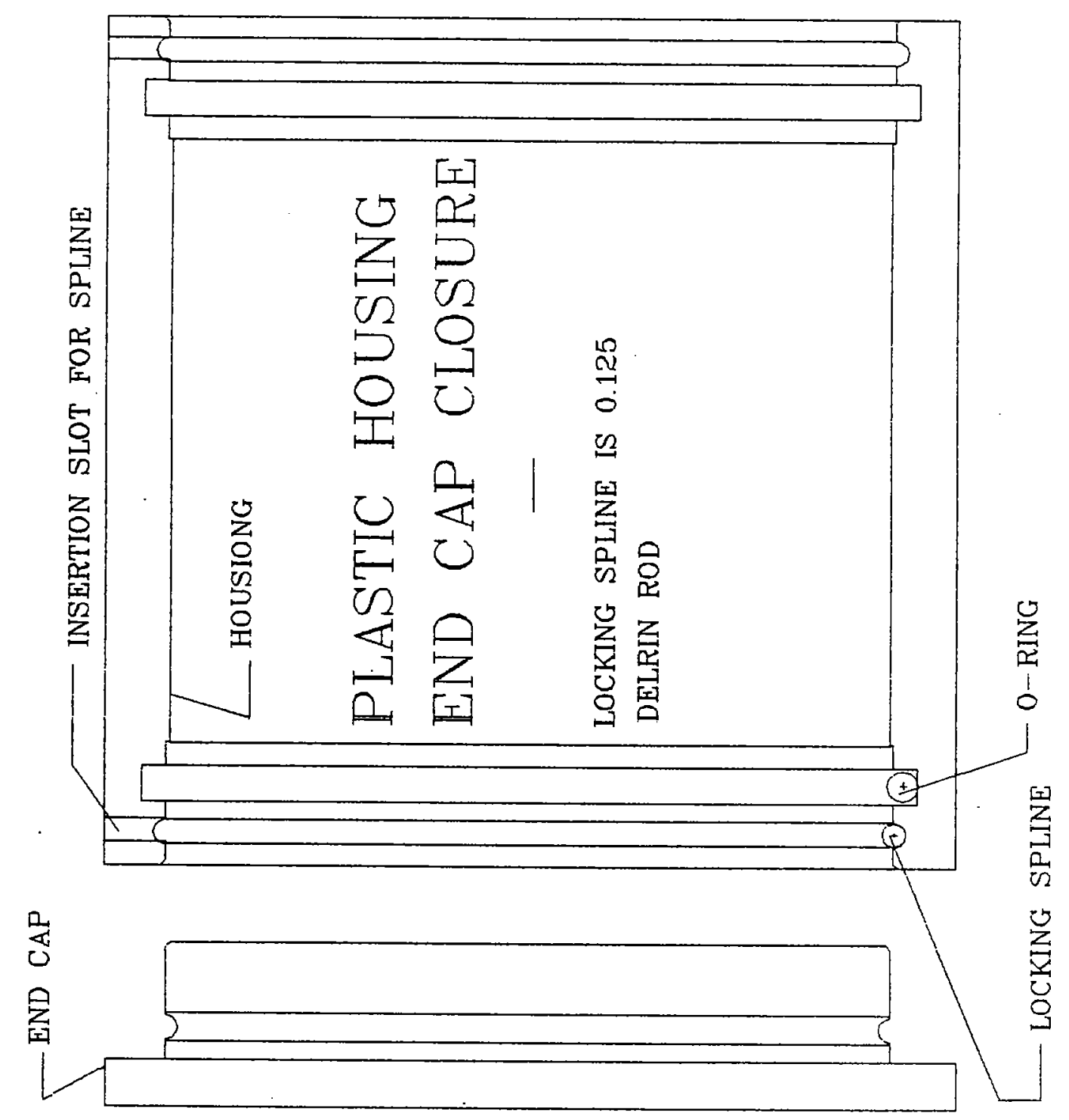

Mechanical detail of IMET Module Closure 


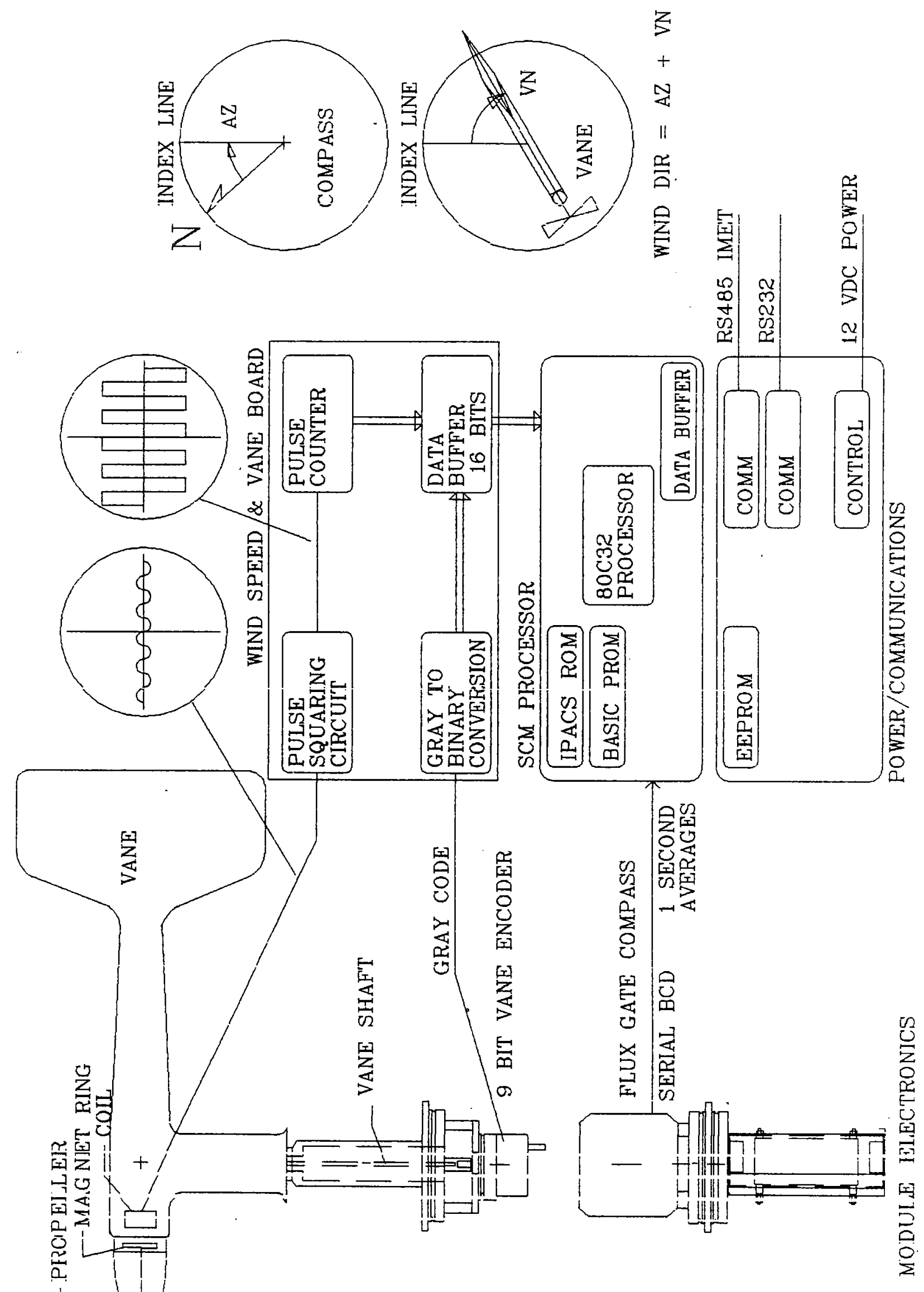

IMET Wind Module Block Diagram 




\section{DOCUMENT LIBRARY}

\section{Distribution List for Technical Report Exchange - May 5, 1994}

University of California, San Diego

SIO Library 0175C (TRC)

9500 Gilman Drive

La Jolla, CA 92093-0175

Hancock Library of Biology \& Oceanography

Alan Hancock Laboratory

University of Southern California

University Park

Los Angeles, CA 90089-0371

Gifts \& Exchanges

Library

Bedford Institute of Oceanography

P.O. Box 1006

Dartmouth, NS, B2Y 4A2, CANADA

Commander

International Ice Patrol

1082 Shennecossett Road

Groton, CT 06340-6095

NOAA/EDIS Miami Library Center

4301 Rickenbacker Causeway

Miami, FL 33149

Library

Skidaway Institute of Oceanography

10 Ocean Science Circle

Savannah, GA 31411

Institute of Geophysics

University of Hawaii

Library Room 252

2525 Correa Road

Honolulu, HI 96822

Marine Resources Information Center

Building E38-320

MIT

Cambridge, MA 02139

Library

Lamont-Doherty Geological Observatory

Columbia University

Palisades, NY 10964

Library

Serials Department

Oregon State University

Corvallis, OR 97331

Pell Marine Science Library

University of Rhode Island

Narragansett Bay Campus

Narragansett, RI 02882
Working Collection

Texas A\&M University

Dept. of Oceanography

College Station, TX 77843

Fisheries-Oceanography Library 151 Oceanography Teaching Bldg.

University of Washington

Seattle, WA 98195

Library

R.S.M.A.S.

University of Miami

4600 Rickenbacker Causeway

Miami, FL 33149

Maury Oceanographic Library

Naval Oceanographic Office

Building 1003 South

1002 Balch Blvd.

Stennis Space Center, MS 39522-5001

Library

Institute of Ocean Sciences

P.O. Box 6000

Sidney, B.C. V8L 4B2

CANADA

Library

Institute of Oceanographic Sciences

Deacon Laboratory

Wormley, Godalming

Surrey GU8 5UB

UNITED KINGDOM

The Librarian

CSIRO Marine Laboratories

G.P.O. Box 1538

Hobart, Tasmania

AUSTRALIA 7001

Library

Proudman Oceanographic Laboratory

Bidston Observatory

Birkenhead

Merseyside L43 7 RA

UNITED KINGDOM

IFREMER

Centre de Brest

Service Documentation - Publications

BP 7029280 PLOUZANE

FRANCE 


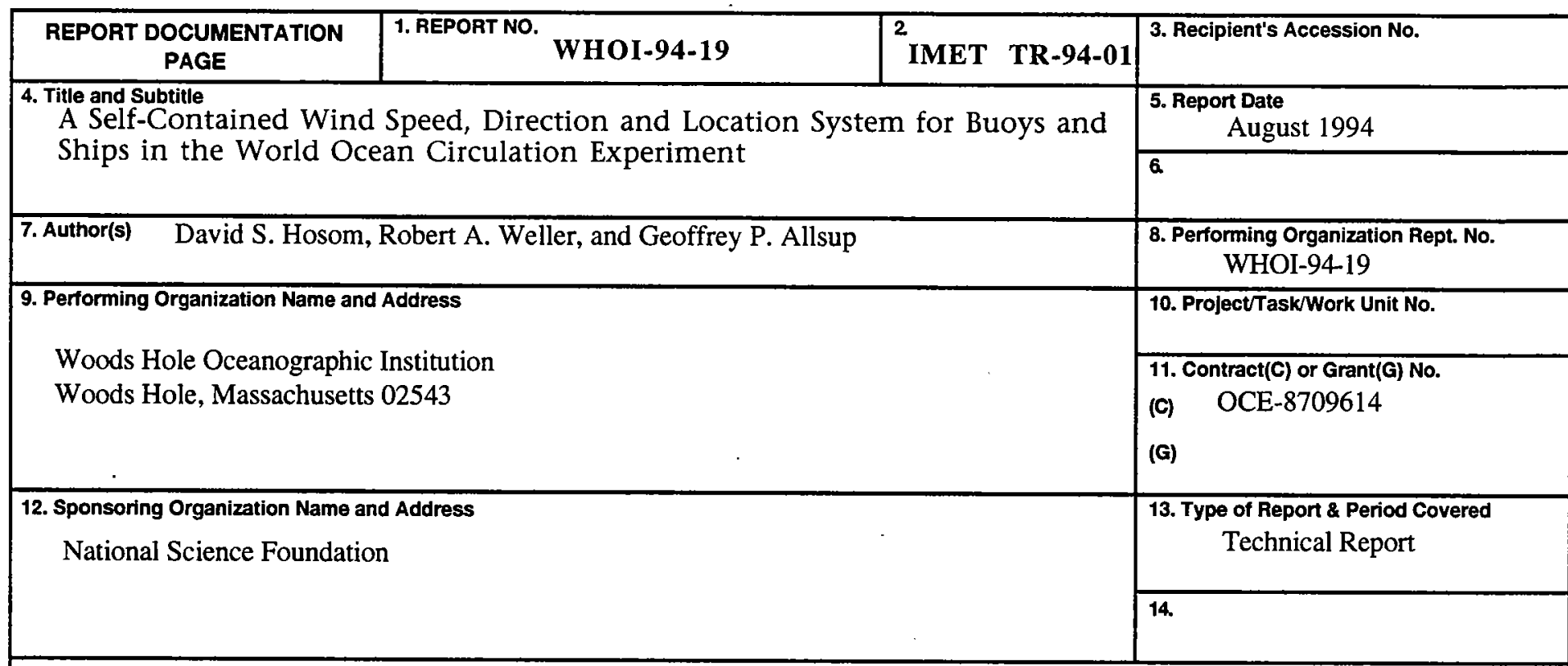

\section{Supplementary Notes}

This report should be cited as: Woods Hole Oceanog. Inst. Tech. Rept., WHOI-94-19.

\section{Abstract (Limit: 200 words)}

Knowledge of the absolute wind velocity near the surface of the ocean is a requirement of the World Ocean Circulation Experiment (WOCE) and other large programs directed towards understanding air-sea interactions and how ocean circulation and climate are interrelated. The measurement is made possible using IMET (Improved METeorology) modules, a next generation meteorological data acquisition system developed as part of the WOCE program. An IMET system consists of a set of intelligent modules for each measurement variable, with data being recorded on a computer, typically PC-based. The IMET wind module includes a propeller for wind speed, a vane and optical encoder for wind direction, a flux gate compass for the north reference, and microprocessor-based electronics for control and data formatting. The IMET Global Positioning System (GPS) module includes a five channel GPS receiver and microprocessor based electronics for control and data formatting. These modules, as part of the complete measurement suite, result in a self-contained system that can make accurate measurements from research ships, drifting and moored buoys, and volunteer observing ships (VOS).

\section{Document Analysis a. Descriptors Global Ocean \\ ECO system \\ Dynamics}

b. Identifiers/Open-Ended Terms

c. COSATI Field/Group

18. Availability Statement

Approved for public release; distribution unlimited.

\begin{tabular}{|l|l|}
\hline $\begin{array}{c}\text { 19. Security Class (This Report) } \\
\text { UNCLASSIFIED }\end{array}$ & $\begin{array}{c}\text { 21. No. of Pages } \\
24\end{array}$ \\
\hline 20. Security Class (This Page) & 22. Price \\
\hline verse & $\begin{array}{l}\text { OPTIONAL FORM 272 (4-77) } \\
\text { (Formerly NTIS-35) } \\
\text { Department of Commerce }\end{array}$ \\
\hline
\end{tabular}


